\title{
ASPECTOS BIOLÓGICOS DO CAMARÃO-ESPINHO Exhippolysmata oplophoroides (HOLTHUIS, 1948) (CRUSTACEA, CARIDEA, HIPPOLYTIDAE).
}

\author{
CHACUR, M. M. e NEGREIROS-FRANSOZO, M. L. \\ Núcleo de Estudos em Biologia, Ecologia e Cultivo de Crustáceos, Departamento de Zoologia, Instituto de \\ Biociências, UNESP, C.P. 510, CEP 18618-000, Botucatu, SP, Brasil \\ Correspondência para: Negreiros-Fransozo, M. L., Núcleo de Estudos em Biologia, Ecologia e Cultivo de \\ Crustáceos, Departamento de Zoologia, Instituto de Biociências, UNESP, C.P. 510, CEP 18618-000, \\ Botucatu, SP, Brasil, e-mail: mlnf@ibb.unesp.br \\ Recebido em 02/09/97 - Aceito em 21/10/98 - Distribuído em 23/02/98
}

(Com 4 figuras)

\section{ABSTRACT \\ Biological aspects of the spine-shrimp Exhippolysmata oplophoroides (Holthuis, 1948) (Crustacea, Caridea, Hippolytidae)}

The number of eggs and the proportion in which they are produced can provide an indication of the species reproductive potential. The objective of this paper was to get basic data on the general biology of E. oplophoroides and, in particular, its fecundity in the area of Ubatuba (SP). The specimens were collected by trawl in the Ubatuba littoral $\left(23^{\circ} \mathrm{S} / 45^{\circ} \mathrm{N}\right)$ from June/1995 to May/1996. The number of eggs found for each female was plotted against to the cephalothoracic lenght and a linear regression was adjusted.

The mean fecundity obtained was $2,742 \pm 1,561$ which varied from 434 to 5,215 eggs per female per spawn and the mean volume of eggs was $0.015 \pm 0.002 \mathrm{~mm}^{3}$. Fecundity increases with the growth of E. oplophoroides females. In the sampled population, it was not found males, suggesting sexual reversion which is very common in marine caridean shrimp. The high percentage of ovigerous females during the whole year confirms the fact of the tropical species present continuous reproduction.

Key words: fecundity, Caridea, Hippolytidae.

\section{RESUMO}

O número de ovos e a taxa na qual eles são produzidos podem fornecer uma indicação do potencial reprodutivo de uma espécie. O objetivo deste estudo foi levantar dados básicos sobre a biologia geral de E. oplophoroides e, em particular, sua fecundidade na região de Ubatuba, SP. Os espécimes foram coletados em arrastos camaroneiros na Enseada de Ubatuba, SP $\left(23^{\circ} \mathrm{S} / 45^{\circ} \mathrm{N}\right)$, no período de junho/1995 a maio/1996.

O número de ovos relacionados a uma dimensão corpórea (comprimento cefalotorácico) foi ajustado a uma regressão linear. A fecundidade média obtida foi de $2.742 \pm 1.561$, variando de 434 a 5.215 ovos por fêmea por desova e o volume médio dos ovos foi de $0,015 \pm 0,002 \mathrm{~mm}^{3}$. A fecundidade aumentou proporcionalmente com o tamanho de E. oplophoroides. Não houve registro de machos no período de estudo, sugerindo uma possível existência de reversão sexual na espécie, visto que tal fato é bastante comum em se tratando de camarões carídeos marinhos. A alta porcentagem de fêmeas ovígeras durante todo o ano confirma o fato das espécies tropicais apresentarem reprodução contínua.

Palavras-chave: fecundidade, Caridea, Hippolytidae. 


\section{INTRODUÇÃO}

A ordem Decapoda compreende um grupo bastante diversificado, cujos representantes possuem múltiplas adaptações a modos de vida muito variados. Sua evolução inclui uma inovação muito importante, única entre os Malacostraca, que é o aparecimento de incubação pleopodial, com eclosão tipicamente no estágio de zoea; este cuidado parental resulta numa grande sobrevivência da prole. De acordo com Saint-Laurent (1979), esse modo de desenvolvimento caracteriza a subordem Pleocyemata, onde está incluída a infraordem Caridea.

O conhecimento da fecundidade, que corresponde ao número de ovos postos por fêmea por desova que se encontram aderidos aos pleópodos (Santos, 1978 e Lobão et al., 1985), pode fornecer uma indicação do desempenho do potencial reprodutivo de uma espécie. Essa informação é muito útil para estudos populacionais e constitui-se em subsídio importante para uma avaliação do potencial da espécie para o cultivo em escala comercial.

O tamanho dos ovos colocados por uma espécie é, em parte, controlada geneticamente, mas também determinada fenotipicamente (Raven, 1961 apud Sastry, 1983). O tamanho dos ovos tem importantes consequiências na taxa de desenvolvimento e no tamanho das larvas ou jovens, no caso de crustáceos com desenvolvimento direto, no momento da eclosão (Negreiros-Fransozo et al., 1992).

A pesca artesanal do camarão sete-barbas, Xiphopenaeus kroyeri, (Heller, 1862), efetuada com barcos providos de rede de arrasto é comum no litoral norte paulista. Essa técnica, além de capturar os camarões peneídeos, captura também outros decápodos entre os quais siris, caranguejos e camarões carídeos.

Entre os carídeos, a espécie Exhippolysmata oplophoroides (Holthuis, 1948) conhecida comummente por camarão-espinho, é uma das mais comuns no nosso litoral. Apesar disso, não há nenhum registro de estudos a respeito do seu ciclo reprodutivo.

O objetivo deste estudo foi levantar dados básicos sobre a biologia geral de E. oplophoroides e, em particular, sua fecundidade na região de Ubatuba (SP).

\section{MATERIAL E MÉTODOS}

A espécie Exhippolysmata oplophoroides apresenta coloração avermelhada, rostro longo, delgado, curvado para cima, porção dorsal contendo uma crista com nove ou dez dentes próximos e um dente um pouco distante da crista, margem dorsal com um a seis dentes e margem ventral com dez a quatorze dentes (Williams, 1984). Segundo esse mesmo autor, a espécie caracteriza-se pela existência de um espinho dorsal no terceiro segmento abdominal.

A distribuição de E. oplophoroides é conhecida como sendo muito extensa: de Cabo Fear River, Carolina do Norte a Port Arkansas, Texas, Guiana Inglesa até a Baía de Santos, vindo misturados com o camarão sete-barbas, em profundidade de menos de vinte metros e fundo de lodo (Iwai, 1983).

Os camarões E. oplophoroides foram capturados durante o dia, mensalmente, na Enseada de Ubatuba, SP, $\left(23^{\circ} 25^{\prime} \mathrm{S} / 45^{\circ} 00^{\prime} \mathrm{W}\right)$ em arrastos efetuados paralelamente a um costão rochoso (sentido Ponta do Costa - Praia Vermelha do Norte), no período de junho/95 a maio/96. Cada coleta constituiu-se de um arrasto, com rede camaroneira, durante 20 minutos a uma velocidade constante. Paralelamente, foram coletados dados de temperatura e salinidade locais. Após o término do arrasto, os camarões da espécie E. oplophoroides foram separados, contados e etiquetados. As fêmeas ovígeras foram separadas e acondicionadas individualmente.

Todos os camarões-espinho coletados foram mensurados com o auxílio de um paquímetro com precisão de décimos de milímetros. Considerouse o comprimento cefalotorácico (CC) como sendo a distância tomada desde a parte posterior da órbita ocular até a parte mediana posterior do cefalotórax (Fig. 1).

Em cada amostra foram selecionados indivíduos com diferentes colorações de gônadas para preparações histológicas, com o intuito de detectar a presença de indivíduos machos. Nesses casos, retirou-se dorsalmente a carapaça dos camarões, os quais foram fixados com Davidson's AFA (Humason, 1972 apud Bell \& Lightner, 1988).

Para o estudo da fecundidade foram selecionadas fêmeas ovígeras de E. oplophoroides com ovos em estágio inicial de desenvolvimento. Os 
pleópodos de cada fêmea foram cortados no ponto de articulação com o abdome e colocados sobre placas de Petri com álcool.

Os ovos foram removidos com o auxílio de um pincel fino. Com uma pipeta, os ovos foram transferidos para uma lâmina de vidro quadriculada e, a seguir, realizou-se a contagem total sob estereomicroscópio óptico.

Dez fêmeas ovígeras foram selecionadas para a mensuração dos ovos. As medidas (diâmetros maior e menor) foram efetuadas sob estereomicroscópio óptico, provido de ocular micrométrica. $\mathrm{O}$ volume dos mesmos foi calculado segundo Jones \& Simons (1983) e Clarke (1993), a partir da fórmula elipsóide $1 / 6 \pi \mathrm{I}^{3}$, em que $\mathrm{I}=$ valor médio dos diâmetros maior e menor.

Vinte e três fêmeas ovígeras foram distribuídas em 9 classes de tamanho de 7,0 a 16,0 mm com $1 \mathrm{~mm}$ de amplitude por classe. A relação entre a fecundidade e a variável morfométrica foi analisada por uma regressão linear simples. Adotouse como variável dependente o logaritmo (na base 10) do número de ovos e variável independente, o comprimento cefalotorácico $(\mathrm{mm})$.

Para verificar uma possível associação entre a porcentagem de fêmeas ovígeras com o fator temperatura durante os meses amostrados, utilizouse o coeficiente de correlação linear de Pearson adotando-se o nível de significância de $5 \%$.

\section{RESULTADOS E DISCUSSÃO}

O local onde foram coletados os camarõesespinho recebe pouca influência de águas continentais, tendo em vista que a área está mais sujeita à influência marinha. A temperatura média anual da água no local de estudo foi de $23,39 \pm 2,86{ }^{\circ} \mathrm{C}$ e a salinidade $33,33 \pm 1,70 \%$. Os valores médios mensais para estes fatores encontram-se na Tabela 1.

Os 560 exemplares de E. oplophoroides obtidos foram distribuídos em 10 classes de tamanho baseando-se no comprimento cefalotorácico. O tamanho dos indivíduos variou de 6,2 a $16,6 \mathrm{~mm}$, com um valor médio de $11,4 \mathrm{~mm}$. A distribuição dos indivíduos em classes de tamanho (Fig. 2), em 2 grupos de interesse (presença ou não de ovos), apresentou-se unimodal, sendo as fêmeas ovígeras encontradas em maior freqüência nas maiores classes de tamanho.

Com base na morfologia externa dos camarões, não se obteve exemplares machos de E. oplophoroides em nenhum dos meses amostrados.

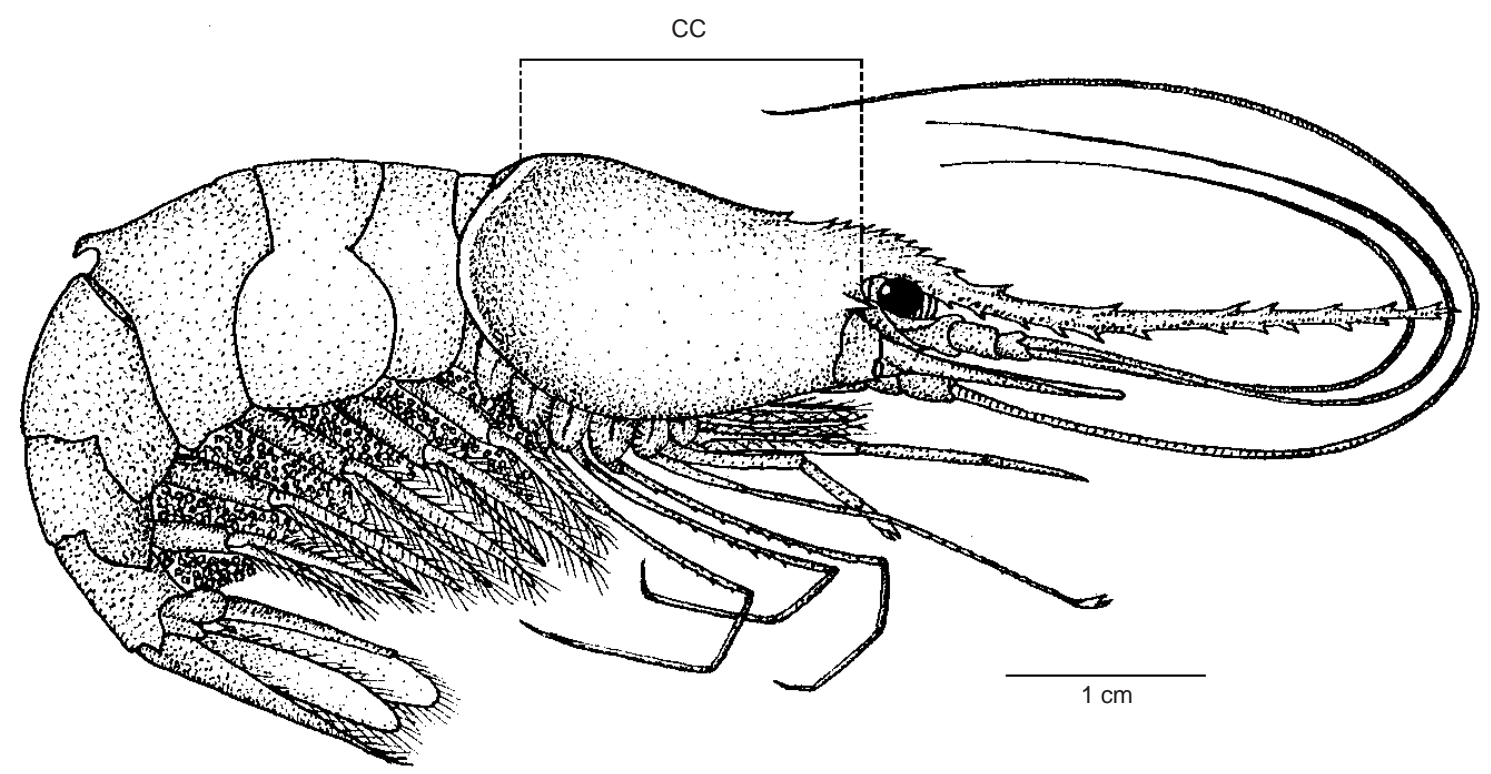

Fig. 1 - E. oplophoroides: exemplar fêmea adulta ovígera com a indicação da dimensão usada na análise morfométrica (CC). 


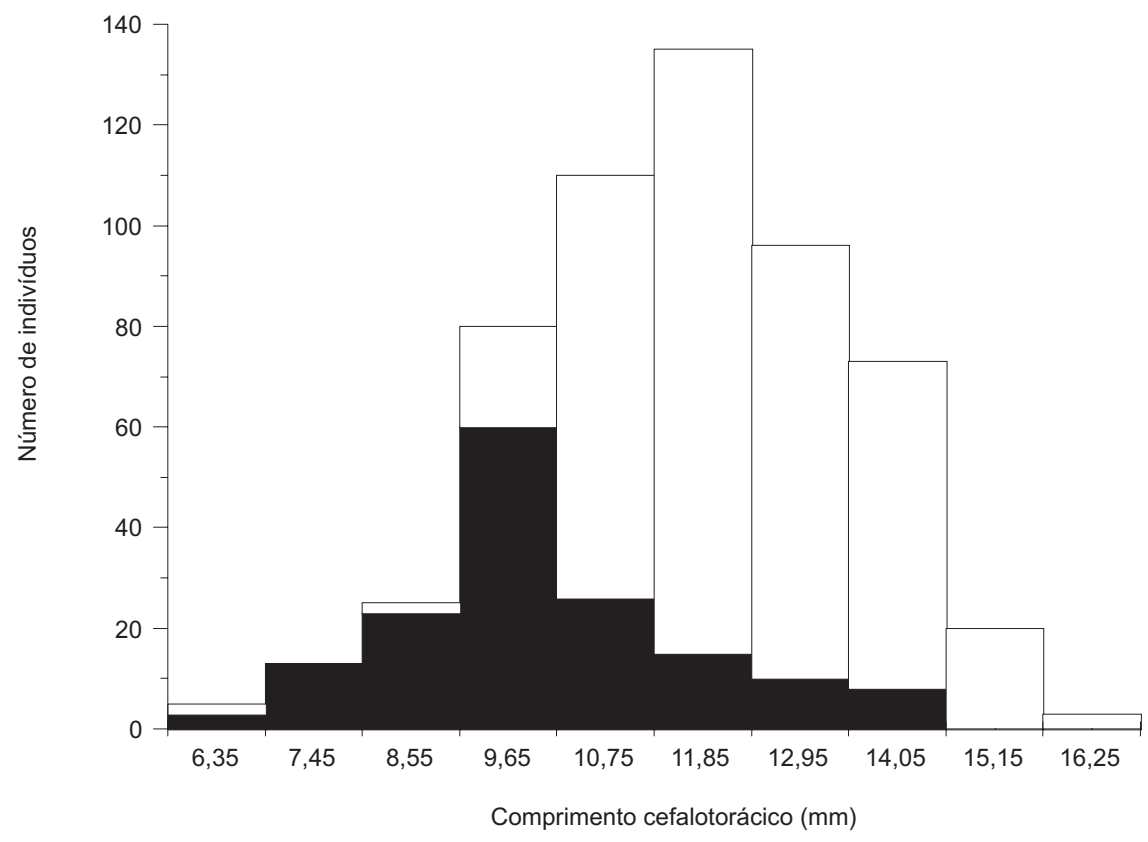

Fig. 2 - E. oplophoroides: histograma de freqüência baseando-se na presença ou não de ovos (barra branca = fêmeas ovígeras; barra preta $=$ outros $)$.

As preparações histológicas, também não indicaram presença de gônadas masculinas.

De aproximadamente 30 espécies de decápodos, nas quais ocorre reversão de sexo (Carpenter, 1978; Subramonian, 1981; Bauer, 1986), mais de $80 \%$ são camarões carídeos, a maioria membros das famílias Campylonotidae, Crangonidae, Hippolytidae, Pandalidae e Processidae (Suzuki, 1970; Noel, 1973; Zupo, 1994).

As mudanças morfológicas externas que ocorrem nos caracteres sexuais secundários, constituem o critério comumente usado para identificar o estágio de transição de sexo em camarões carídeos. O endopodito do $1^{\circ}$ pleópodo se modifica gradualmente para a forma lanceolada das fêmeas; o endopodito do $2^{\circ}$ pleópodo, o apêndice masculino e órgãos copulatórios, degeneram até essas estruturas serem totalmente perdidas nas fêmea (Allen, 1959; Haynes \& Wigley, 1969; Butler, 1964). No entanto, esse critério não foi suficiente para identificar tais estágios em E. oplophoroides.

A falta de indivíduos masculinos na população amostrada e a predominância de fêmeas portadoras de ovos pode ser conseqüência da existência de reversão sexual em E. oplophoroides, visto que este processo pode ocorrer em outras espécies de carídeos. No entanto, a possibilidade de uma distribuição espacial diferencial entre os sexos não pode ser descartada. Para se chegar a uma conclusão satisfatória são necessários estudos mais detalhados sobre a distribuição da espécie na região de estudo, além de uma análise histológica pormenorizada das gônadas.

No estudo da fecundidade, a menor fêmea ovígera apresentou comprimento cefalotoráxico medindo $7,2 \mathrm{~mm}$ e a maior $15,7 \mathrm{~mm}$, com tamanho médio de $11,51 \pm 2,48 \mathrm{~mm}$. O número médio de ovos encontrado foi $2.742 \pm 1.561$ com variação de 434 a 5.215 ovos por fêmea (Tabela 2).

O número de ovos carregados por um crustáceo Pleocyemata é relativamente variável para uma mesma classe de tamanho (Lobão et al., 1985; Negreiros-Fransozo et al., 1992), conforme registrado no presente estudo. Segundo Parsons \& Tucker (1986), tal fato deve-se à perda acidental dos ovos ou devido à fertilização incompleta ou, ainda, a doenças.

A relação do logaritmo do número de ovos postos por fêmea em função do comprimento cefalotorácico está representada na Fig. 3, a partir da qual conclui-se que a fecundidade eleva-se com o aumento em comprimento em E. oplophoroides. 
Tal fato ocorre em outros carídeos como o Macrobrachium carcinus, M. potiuna, M. acanthurus e Hippolyte zostericola estudados, respectivamente, por Lobão et al. (1985), Bond \& Buckup (1982), Valenti et al. (1989) e Negreiros-Fransozo et al. (1996).

Clarke (1993) afirma que o tamanho dos ovos dos invertebrados aquáticos varia amplamente, refletindo uma variação do investimento pela fêmea em cada embrião e as fêmeas menores desovam menor número de ovos, por apresentarem um ovário menor e um reduzido espaço no abdome para a incubação dos mesmos. Entre os decápodos, verifica-se que os carídeos apresentam um número pequeno de ovos, porém, estes crustáceos figuram entre os que possuem ovos relativamente grandes.

TABELA 1

Valores médios mensais e desvio-padrão dos fatores temperatura e salinidade na Enseada de Ubatuba no período de junho de 1995 a maio de 1996.

\begin{tabular}{lcc}
\hline Mês & Temperatura $\left({ }^{\mathbf{0}} \mathbf{C}\right)$ & Salinidade (ppm) \\
\hline Junho & $23,0 \pm 0,04$ & $34,3 \pm 0,47$ \\
Julho & $20,2 \pm 0,10$ & $35,3 \pm 0,47$ \\
Agosto & $20,0 \pm 0,02$ & $33,8 \pm 0,75$ \\
Setembro & $22,2 \pm 0,42$ & $32,7 \pm 0,94$ \\
Outubro & $22,2 \pm 0,17$ & $32,5 \pm 0,41$ \\
Novembro & $22,5 \pm 0,42$ & $30,0 \pm 0,82$ \\
Dezembro & $20,3 \pm 1,72$ & $31,5 \pm 0,41$ \\
Janeiro & $26,4 \pm 0,32$ & $35,3 \pm 0,47$ \\
Fevereiro & $27,5 \pm 0,39$ & $31,7 \pm 0,47$ \\
Março & $28,2 \pm 0,12$ & $34,3 \pm 0,25$ \\
Abril & $25,7 \pm 0,30$ & $35,3 \pm 0,25$ \\
Maio & $22,5 \pm 0,98$ & $33,3 \pm 0,94$
\end{tabular}

TABELA 2

E. oplophoroides: fecundidade registrada para cada classe de tamanho.

\begin{tabular}{ccc}
\hline $\begin{array}{c}\text { Comprimento } \\
\text { cefalotorácico }(\mathbf{m m})\end{array}$ & Número de ovos & Número de fêmeas analisadas \\
\hline $7--] 8$ & $664 ; 860$ & 2 \\
$8--] 9$ & $434 ; 864$ & 2 \\
$9--] 10$ & $1.167 ; 814 ; 1.939$ & 3 \\
$10--] 11$ & $1.910 ; 1.903 ; 1.474$ & 3 \\
$11--] 12$ & $3.259 ; 3.561 ; 3.262$ & 3 \\
$12--] 13$ & $3.460 ; 3.998 ; 5.100$ & 3 \\
$13--] 14$ & $4.202 ; 5.165 ; 2.462$ & 2 \\
$14--] 15$ & $5.215 ; 3.186$ & 2 \\
\hline
\end{tabular}


A forma dos ovos de E. oplophoroides é elíptica, sendo os resultados da morfometria apresentados na Tabela 3. Para a espécie em estudo, o tamanho dos ovos num mesmo estágio embriônico não variou com o tamanho da fêmea.

Entre as espécies da super família Alpheoidea estudadas anteriormente, a espécie E. oplophoroides apresentou maior número de ovos e menor volume dos mesmos (Tabela 4). Este fato pode ser um importante fator na definição da extensão do desenvolvimento embrionário, quer seja no número de estágios, quer seja no tempo requerido para completar a metamorfose. A presença de fêmeas ovígeras durante todo o ano, evidenciou um alto potencial reprodutivo de E. oplophoroides. Não se obteve correlação significativa $(r=0,545$; $\mathrm{p}=0,067)$ entre a taxa de fêmeas ovígeras e a temperatura da água evidenciando que a variação da temperatura da água no local estudado não se apresentou limitante à reprodução da espécie.

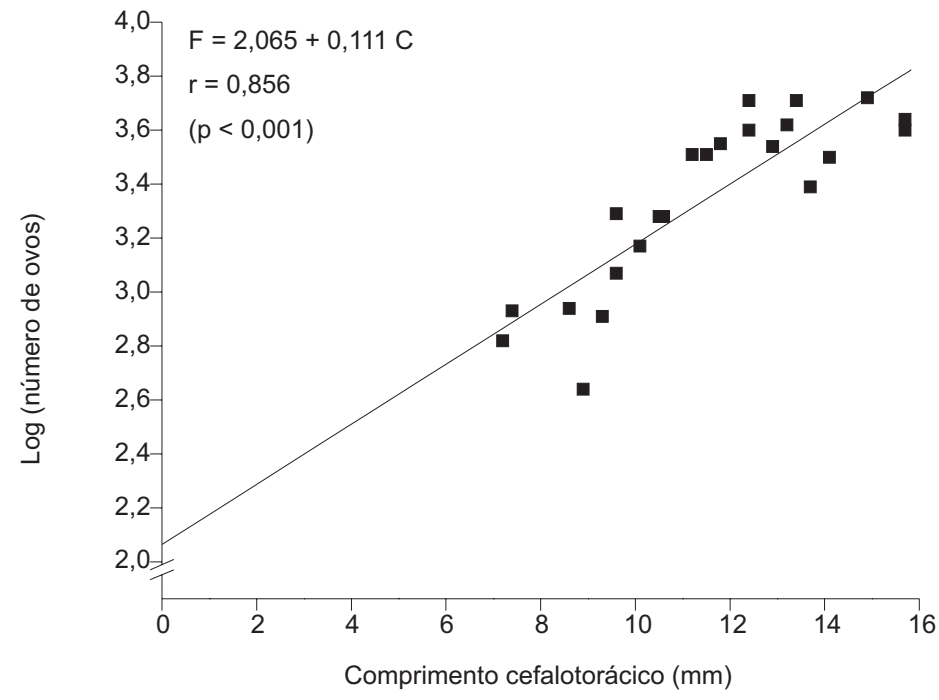

Fig. 3 - E. oplophoroides: gráfico da regressão linear entre o log do número de ovos (F) e o comprimento cefalotorácico (C).

TABELA 3

E. oplophoroides: Diâmetros médios e volume dos ovos em estágio inicial de desenvolvimento, em relação ao tamanho das fêmeas.

\begin{tabular}{cccc}
\hline $\begin{array}{c}\text { Comprimento } \\
\text { cefalotorácico }(\mathbf{m m})\end{array}$ & $\begin{array}{c}\text { Diâmetro maior } \\
(\mathbf{m m})\end{array}$ & $\begin{array}{c}\text { Diâmetro menor } \\
(\mathbf{m m})\end{array}$ & $\begin{array}{c}\text { Volume dos ovos } \\
\left(\mathbf{m m}^{\mathbf{3}}\right)\end{array}$ \\
\hline 7,3 & $0,324 \pm 0,033$ & $0,268 \pm 0,033$ & 0,015 \\
9,9 & $0,366 \pm 0,001$ & $0,293 \pm 0,027$ & 0,019 \\
10,0 & $0,329 \pm 0,033$ & $0,256 \pm 0,027$ & 0,015 \\
10,3 & $0,342 \pm 0.033$ & $0,268 \pm 0,033$ & 0,015 \\
11,2 & $0,342 \pm 0,033$ & $0,256 \pm 0,027$ & 0,014 \\
11,6 & $0,329 \pm 0,033$ & $0,256 \pm 0,027$ & 0,013 \\
11,6 & $0,354 \pm 0,027$ & $0,281 \pm 0,033$ & 0,017 \\
12,2 & $0,317 \pm 0,027$ & $0,244 \pm 0,001$ & 0,011 \\
13,2 & $0,342 \pm 0,033$ & $0,268 \pm 0,033$ & 0,015 \\
15,2 & $0,354 \pm 0,027$ & $0,256 \pm 0,027$ & 0,015 \\
\hline
\end{tabular}


TABELA 4

Comparação da fecundidade de camarões carídeos.

\begin{tabular}{|c|c|c|c|c|c|}
\hline Superfamília & Família & Espécie & $\begin{array}{l}\text { Número de } \\
\text { ovos }\end{array}$ & $\begin{array}{c}\text { Volume dos } \\
\text { ovos } \\
\left(\mathbf{m m}^{\mathbf{3}}\right)\end{array}$ & Autor \\
\hline \multirow[t]{16}{*}{ Alpheoidea } & Hippolytidae & Exhippolysmata oplophoroides & 2.742 & 0,015 & Presente trabalho \\
\hline & & Hippolyte nicholsoni & 24,75 & 0,018 & Corey \& Reid, 1991 \\
\hline & & Hippolyte zostericola & 103,83 & 0,037 & $\begin{array}{l}\text { Negreiros-Fransozo } \\
\text { et al., } 1996\end{array}$ \\
\hline & & Latreutes fucorum & 126,84 & 0,030 & Corey \& Reid, 1991 \\
\hline & & Thor manningi & 37,14 & 0,036 & Corey \& Reid, 1991 \\
\hline & & Tozeuma carolinense & 190,16 & 0,057 & Corey \& Reid, 1991 \\
\hline & & Trachycaris restricta & 431,25 & 0,220 & Corey \& Reid, 1991 \\
\hline & Alpheidae & Alpheus armillatus & 279,75 & 0,172 & Corey \& Reid, 1991 \\
\hline & & A. heterochaelis & 203,40 & 0,912 & Corey \& Reid, 1991 \\
\hline & & A. normanni & 328,14 & 0,094 & Corey \& Reid, 1991 \\
\hline & & Synalpheus aeglas & 42,4 & 0,228 & Corey \& Reid, 1991 \\
\hline & & S. brooksi & 5,8 & 0,504 & Corey \& Reid, 1991 \\
\hline & & S. fritzmueller & 173,38 & 0,085 & Corey \& Reid, 1991 \\
\hline & & S. herricki & 45,75 & 0,222 & Corey \& Reid, 1991 \\
\hline & & S. longicarpus & 195,38 & 0,169 & Corey \& Reid, 1991 \\
\hline & & S. pectininger & 9,9 & 0,751 & Corey \& Reid, 1991 \\
\hline \multirow[t]{13}{*}{ Palaemonoidea } & Palaemonidae & Leander tenuicornis & 452,08 & 0,163 & Corey \& Reid, 1991 \\
\hline & & Macrobrachium acanthurus & $8.929,00$ & _- _ & Valenti et al., 1989 \\
\hline & & M. carcinus & $53.764,00$ & _- & Lobão et al., 1985 \\
\hline & & M. ohione & $11.050,5$ & 0,080 & Corey \& Reid, 1991 \\
\hline & & M. olfersii & $3.098,88$ & 0,076 & Corey \& Reid, 1991 \\
\hline & & Palaemon northropi & 395,23 & 0,200 & Corey \& Reid, 1991 \\
\hline & & Palaemonetes intermedius & 104,86 & 0,294 & Corey \& Reid, 1991 \\
\hline & & P. pugio & 109,62 & 0,320 & Corey \& Reid, 1991 \\
\hline & & Pandalopsis dispar & 904 & _- _ & Harris et al., 1972 \\
\hline & & Periclimenes americanus & 247,33 & 0,034 & Corey \& Reid, 1991 \\
\hline & & P. longicaudatus & 92,75 & 0,049 & Corey \& Reid, 1991 \\
\hline & & P. pandionis & 171,00 & 0,050 & Corey \& Reid, 1991 \\
\hline & & Pontonia margarita & $1.538,25$ & 0,093 & Corey \& Reid, 1991 \\
\hline Pandaloidea & Pandalidae & Dichelopandalus bonnieri & $1.400,5$ & - _- & Al-Adhub \& Bowers, 1977 \\
\hline
\end{tabular}


Como pode ser observado na Fig. 4, a menor taxa de fêmeas ovígeras não foi inferior a $50 \%$. Tal fato confirma a proposição feita por Sastry (1983), na qual a maioria das espécies tropicais apresenta reprodução contínua.
Agradecimentos - Ao Conselho Nacional de Desenvolvimento Científico e Tecnológico (CNPq), pela concessão da bolsa de estudos de aperfeiçoamento à primeira autora; aos membros do NEBECC, pelo auxílio nas coletas, e ao assessor anônimo da Revista Brasileira de Biologia, pelas valiosas sugestões ao manuscrito.

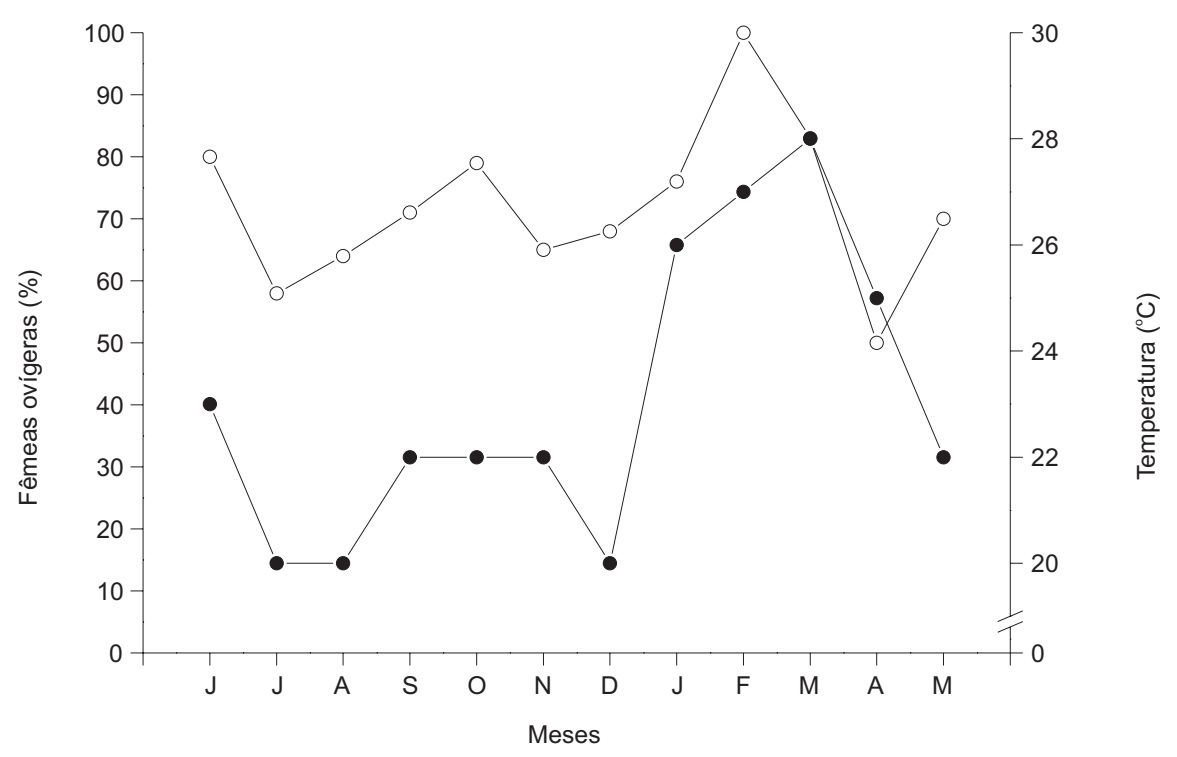

Fig. 4 - Gráfico de linhas da taxa de fêmeas ovígeras e da temperatura ao longo dos meses de coleta (círculo aberto = fêmeas ovígeras; círculo fechado = temperatura).

\section{REFERÊNCIAS BIBLIOGRÁFICAS}

AL-ADHUB, A. H. \& BOWERS, C. A. B., 1977, Growth and breeding of Dichelopandalus bonnieri in isle of man waters. J. Mar. Biol. Ass. U. K., 57: 229-238.

ALLEN, J. A., 1959, On the biology of Pandalus borealis umderland coast. J. Mar. Biol. Assoc. U. K., 38: 189-220.

BAUER, R. T., 1986, Sex change and life history pattern in the shrimp Thor manningi (Decapoda: Caridea): a novel case of partial protandric hermaphroditism. Biol. Bull., 170: $11-31$

BELL, T. A. \& LIGHTNER, D. V., 1988, A handbook of normal penaeid shrimp histology. Louisiana: World Aquaculture Society/ Baton Rouge, 114p.

BOND, G. \& BUCKUP, L., 1982, O ciclo reprodutivo de $M a-$ crobrachium borellii (Mobilii, 1896) e Macrobrachium potiuna (Müller, 1880) (Crustacea, Decapoda, Palaemonidae) e suas relações com a temperatura. Rev. Brasil. Biol., 42(3): 473-483.

BUTLER, T. H., 1964, Growth, reproduction and distribution of pandalid shrimp in British Columbia. J. Fish. Res. Board. Can., 21: 1403-1452.
CARPENTER, A., 1978, Protandry in freshwater shrimp Paratya curviostris (Heller, 1862) (Decapoda: Atyidae), with a review of the phenomenon and its significance in the Decapoda. J. Res. Soc. N. Z., 8: 343-358.

CLARKE, A., 1993, Reproductive trade-offs in caridean shrimps. Functional Ecology, 7: 411-419.

COREY, S. \& REID, D. M., 1991, Comparative fecundity of Decapod Crustaceans 1 . The fecundity of thirty-three species of nine families of caridean shrimp. Crustaceana, 60: $270-294$.

HARRIS, C., CHEW, K. K. \& PRICE, V., 1972, Relation of egg number to carapace lenght of sidestrip shrimp (Pandalopsis dispar) from Dabob Bay, Washington. J. Fish. Res. Bd. Canada, 29(4): 464-465.

HAYNES, E. B. \& WIGLEY, R. L., 1969, Biology of the northern shrimp, Pandalus borealis, in the Gulf of Marine. Trans. Am. Fish. Soc., 98: 60-76.

IWAI, M., 1983, O camarão no Centro Sul do Brasil. São Paulo: Superintendência do Desenvolvimento do Litoral Paulista, Instituto Oceanográfico da Universidade de São Paulo, São Paulo, 71p. 
JONES, M. B. \& SIMONS, M. J., 1983, Latitudinal variation in reproductive characteristics of a mud crab Helice crassa (Grapsidae). Bull. Mar. Sci., 33: 656-670.

LOBÃO, V. L., VALENTI, W. C. \& MELLO, J. T. C., 1985 , Fecundidade em Macrobrachium carcinus, (L.) do Rio Ribeira do Iguape. Bol. Inst. Pesca, 12: 1-8.

NEGREIROS-FRANSOZO, M. L., BARBA, E., SANCHEZ A. J., FRANSOZO, A. \& RAZ-GUZMÁN, A., 1996, The species of Hippolyte Leach (Crustacea, Caridea, Hippolytidae) from Terminos Lagoon, S. W Gulf of Mexico. Rev. Bras. Zool., 13(3): 539-551.

NEGREIROS-FRANSOZO, M. L., FRANSOZO, A. MANTELATTO, F. L. M., NAKAGAKI, J. M. \& SPILBORGHS, M. C. F., 1992, Fecundity of Paguristes tortugae Schmitt, 1933 (Crustacea, Decapoda, Anomura) in Ubatuba (SP), Brazil. Rev. Brasil Biol., 52: 547-553.

NOEL, P., 1973, Cycle biologique et inversion sexuelle du Crustacé Décapode Natantia Processa edulis. Cah. Biol. Mar., 14: 217-227.

PARSONS, D. G. \& TUCKER, G. E., 1986, Fecundity of northern shrimp, Pandalus borealis (Crustacea, Decapoda), in areas of northwest Atlantic. Fish Bull, (Dublin), 84: 549-558.

SAINT-LAURENT, M., 1979, Ver une nouvelle classification des Crustacés Décapodes Reptantia. Bull. Off. Natn. Pêch. Tunisie, 3: 15-31.
SANTOS, E. P., 1978, Dinâmica de populações aplicada à pesca e à piscicultura. São Paulo: HUCITEC/EDUSP, $130 \mathrm{p}$.

SASTRY, A. N., 1983, Ecological aspects of reproduction, pp. 179-270. In: F. J.Vernberg, W. B. Vernberg (eds.), The Biology of Crustacea, vol. 8, 383p. Environmental adaptations, New York, Academic Press.

SUBRAMONIAN, J., 1981, Sexual and reproductive endocrinology of Crustacea. J. Sci. Ind. Res., 40: 396-403.

SUZUKI, H., 1970, Taxonomic review of four alpheid shrimps belonging to the genus Athanas, with reference to their sexual phenomena. Sci. Reports of theYokohama National University, section II, 17: 1-37.

VAlENTI, W. C., MELlo, J. de T. C. \& LOBÃO, V. L., 1989, Fecundidade em Macrobrachium acanthurus (WIEGMAN, 1836) do Rio Ribeira do Iguape (Crustacea, Decapoda, Palaemonidae). Rev. Bras. Zool., 6(1): $9-15$.

WILLIAMS, A. B., 1984, Shrimps, lobsters and crabs of the Atlantic Coast of the Eastern United States, Maine to Florida. Washington: Smithsonian Institution Press, $550 \mathrm{p}$.

ZUPO, V., 1994, Strategies of sexual inversion in Hippolyte inermis Leach (Crustacea, Decapoda) from a Mediterranean seagrass meadow. J. Mar. Biol. Ecol., 178: 131-145. 